\title{
Immunotherapy options for cancer
}

\begin{abstract}
Cancer is a heterogeneous disease originated from various parts of the body and characterized mainly based upon the type of cells or tissue it originated from. In early years of cancer research, it was considered that a "magic drug" can be developed to treat all types of cancers. However, this notion was proven wrong through years of research as various forms of cancer emerged that are variant between individuals and are hard to treat or even control. Clonal survival of cancer cells is the major hurdle limiting the therapeutic response in patient. Cancer progression leads to immunocompromised state in patients resulting in fast disease progression and higher susceptibility to infections. Tumor infiltrated $\mathrm{T}$ cells, tumor associated macrophages, mesenchymal stem cells and other immune cells support the clonal development in cancer tissue leading to its rapid growth and metastasis. In recent years, immunotherapy of cancer is the major interest among researchers. Chimeric antigen receptor (CAR) T cell therapy, immune check point targeting, or boosting the immune system in patient are the approaches being implemented in cancer immunotherapy. Present review discusses the various immunotherapy options for cancer and explains importance of considering the treatment/prevention with natural compounds.
\end{abstract}

Keywords: cancer, immunotherapy, PD1, CTLA4, vitamins, antibodies
Volume 3 Issue 4 - 2017

\author{
Suryatheja Ananthula,' Ashraf Uddin \\ Chowdhury $^{2}$ \\ 'University of Mississippi Medical Center, Cancer Institute, USA \\ ${ }^{2}$ Department of Systems Immunology, Kangwon National \\ University, South Korea
}

Correspondence: Ashraf Uddin Chowdhury, Department of Systems Immunology, College of Biomedical Sciences, Kangwon National University, South Korea, Email ashraf.rx@gmail.com

Received: June 21, 2017 | Published: July 05, 2017
Abbreviations: CAR, chimeric antigen receptor; TAMs, tumor associated macrophages; TNF- $\alpha$, tumor necrosis factor- $\alpha$; VCAM-1, vascular cell adhesion molecule 1; MDSCs, myeloid derived suppressor cells; NK, natural killer; $\mathrm{HER}_{2}$, human epidermal growth factor receptor 2; HLA, human leukocyte antigen; GM-CSF, granulocyte-macrophage colony stimulating factor; $\mathrm{MHC}$; APCs, antigen presenting cells; PBMCs, peripheral blood mononuclear cells; CTLA $_{4}$, cytotoxic t lymphocyte-associated antigen 4

\section{Introduction}

Immune system in the human body comprises of various types of cells, organs, and antibodies including lymphocytes, leukocytes, thymus, lymph nodes, spleen etc. The major function of the immune system is to defend the body against bacteria, virus, antigens and cancer cells. ${ }^{1,2}$ However, cancer cells are a real challenge as they are able to escape immune attack and further immune system favours the tumor progression by altering tumor microenvironment. ${ }^{3}$ Initial research was concentrated on identifying cancer cell gene abnormalities, their transformation, gain/loss of functional mutations, stromal differences and dominant oncogenes. Therapies were developed accordingly which led to partial success in cancer treatment. ${ }^{4-6}$ In many cases, disease free state is being achieved in very few cases and is transient in many patients as the treatment is ineffective or not adequate. ${ }^{7,8}$ There is increasing evidence to suggest that cancer cells also alter and restrict the surrounding normal cells, immune cells and extra cellular components in favour of them. ${ }^{9,10}$ Infiltrated immune cells and tumor associated macrophages (TAMs) can trigger sustained proliferative signals to support cancer cell survival and evolution even in adverse conditions. ${ }^{11}$ Myeloid derived cells and lymphoid cells are complex in nature depending upon the organ they are present in. After infiltrating into the tumours, they can provide growth factors such as tumor necrosis factor $-\alpha(\mathrm{TNF}-\alpha)$, fibroblast growth factor, interleukins, and chemokines to support growth/survival of cancer cells and neighbouring stromal cells in direct and indirect pathways. ${ }^{12}$ Normal tissue cells maintain tissue integrity by their organized cell-to-cell contacts and this function is lost in cancer cells. In this case, tumor cells bind to the immune cells from their surroundings and survive in adverse conditions by promoting growth signals and inhibiting death signals. This type of binding is evident in metastatic breast cancer cells where the $\alpha_{4}$-integrin/VCAM-1 interaction between the tumor associated macrophages and cancer cells respectively induces $\mathrm{PI} 3 \mathrm{~K} /$ Akt pathway to in favour of cell survival. ${ }^{13,14}$ Moreover, immune cells secrete regulatory molecules such as vascular endothelial growth factor, $\mathrm{TNF}-\alpha$, platelet derived growth factors, matrix metalloproteinases and several factors to stimulate vascular cell proliferation and further favour the tumor by remodeling the microenvironment. ${ }^{15}$ Tumor cells alter the functional properties of regulatory $\mathrm{T}$ cells, myeloid derived suppressor cells (MDSCs), TAMs, and natural killer (NK) cells in order to protect themselves from cytotoxic $\mathrm{T}$ cells responses. All these interactions lead to clonal differences in tumor itself. Hence, it is important to study about all the neighbouring cells in tumor region and investigate how the interactions and further actions are being manipulated. Immune check point blockade, cancer vaccines, $\mathrm{T}$ cell based therapies, understanding the micro-biome in regulating cancer therapy, identification of tumor specific and systemic biomarkers are some of the latest strategies in oncoimmunotherapy. ${ }^{16-18}$

Because of increased cancer occurrence worldwide and lack of complete treatment for various cancer types with no adverse effects, it is the time to look into an alternative way to prevent or treat cancer. In an effort, since many years researchers are working on natural compounds in identifying their potent anticancer activities. ${ }^{19}$ several compounds from plant and animal origins are showing promising results in mice and human studies. These natural compounds have an advantage of low or no treatment associated toxicities and can be taken in form of diet on regular basis for prevention of disease occurrence. ${ }^{20-22}$ Some of the natural compounds also have immune modulating activity. ${ }^{23,24}$ Current review explains various options available for cancer immunotherapy along with a brief account of natural compounds tested so far for the same. 


\section{Cancer vaccines}

Vaccines are preparations consisting of a specific antigen and provide altered protective response against a disease. Antigens are prepared to resemble tumor associated antigens or biomarkers and administered to individual with or without adjuvants before the strike of the particular tumor. This helps to boost host immune system to fight against the cancer cells at very early stages of cancer occurrence and further prevents its establishment and growth. ${ }^{25}$ For example, many of the breast cancers show increased levels of HER expression $_{2}$ on cancer cells. Treating this with HER ${ }_{2}$ antibodies is promising in control of the cancer but is associated with significant side effects, tumor relapse or metastasis in majority of the patients. Hence it is advisable to prevent the occurrence by immunizing an individual with antigens designed to resemble $\mathrm{HER}_{2}{ }^{26}$ This particular antigen containing vaccines enhance adaptive immunity in most breast cancer patients. $\mathrm{E}_{75}$ (nelipepimut-S) is a peptide derived from $\mathrm{HER}_{2}$ and is immunogenic in human leukocyte antigen (HLA)- $\mathrm{A}_{2} / \mathrm{A}_{3}$ manner. It was tested in phase I/II clinical trials in breast cancer patients to test its ability to prevent tumor recurrence. ${ }^{27}$ Granulocyte-macrophage colony stimulating factor (GM-CSF) was used as the adjuvant in vaccine preparation from $\mathrm{E}_{75}$. The experiment was conducted in node-positive and high-risk node-negative patients with varying expression levels of $\mathrm{HER}_{2}$. Results indicate $89.7 \%$ disease free survival in vaccinated group of patients while the percentage is $80.2 \%$ is control group of patients. Further, vaccination does not also have significant local or systemic toxicities. ${ }^{27}$ similarly; $\mathrm{AE}_{36}$ is a $\mathrm{HER}_{2}$ derived peptide that can stimulate $\mathrm{CD}_{4+} \mathrm{T}$ cell responses in cancer patients by interacting with $\mathrm{MHC}$ class II molecules. In order to increase $\mathrm{AE}_{36}$ binding efficiency with MHC class II receptor, Ii-key peptide was added to it and together denoted as $\mathrm{AE}_{37}$. Administration of $\mathrm{AE}_{37}$ peptide containing vaccine increases peptide specific proliferation of $\mathrm{CD}_{4+} \mathrm{T}$ cells and IFN $\gamma$ release for cytotoxic T cell activity. ${ }^{28}$ In Phase I clinical trial in breast cancer patients, $\mathrm{AE}_{37}$ was mixed with $\mathrm{GM}-\mathrm{CSF}$ adjuvant and administered to patients in a series of intradermal injections. Grade 1 local toxicities were observed in $40 \%$ of patients and grade 2 levels of toxicities were observed in $60 \%$ of patients. However, adjusting the dose of GM-CSF decreased grade 2 level toxicity. From phase II trials it was proven that $\mathrm{AE}_{37}$ vaccination associated toxicities were well tolerated and safe in patients and induces strong $\mathrm{CD}_{4+} \mathrm{T}$ cell response specific to the peptide. $\mathrm{AE}_{37}$ vaccination also reduced recurrence. ${ }^{28}$

In an alternative way, vaccines are given by loading into cells. Dendritic cells (Antigen presenting cells-APCs) are mainly used to load tumor associated antigens and administration of the same directly presents antigens to immune system in the tumor microenvironment or body for powerful immune response against cancer. ${ }^{29,30}$ Lapuleucel-T is the antigen prepared by loading into peripheral blood mononuclear cells (PBMCs). It consists of HER 2 similar sequence attached to GM-CSF. Lapuleucel-T administration in breast cancer patients was associated with $\mathrm{HER}_{2}$ specific $\mathrm{T}$ cell responses with lower levels of adverse events. ${ }^{31-33}$ Other than $\mathrm{HER}_{2}$ vaccines, $\mathrm{P}_{53}$ specific antigens were also developed to load into dendritic cells. Because $\mathrm{P}_{53}$ is tumor suppressor gene and the mutations in $\mathrm{P}_{53}$ leads to aggressive forms of cancer. ${ }^{34,35}$

\section{PDI/CTLA4 targeted therapy}

Whenever the immune system is activated in the body, check points are also activated after the immune response reached to certain level to inhibit or subdue the immune response in order to prevent any possible organ damage. Programmed death-1 (PD-1) and cytotoxic T lymphocyte-associated antigen $4\left(\right.$ CTLA $\left._{4}\right)$ are the surface check point molecules. ${ }^{36-38} \mathrm{~T}$ cells activation requires the binding of $\mathrm{B}_{7}$ with $\mathrm{CD}_{28}$ on cell surface after the antigen recognition by $\mathrm{T}$ cell receptor. ${ }^{36}$ However, being the homologue of $\mathrm{CD}_{28}, \mathrm{CTLA}_{4}$ bind to $\mathrm{B}_{7}$ and prevents activation signals. $\mathrm{PD}_{1}$ and/or $\mathrm{CTLA}_{4}$ targeted therapy is termed as check point blockade therapy where the antibodies against $\mathrm{PD}_{1}$ and $\mathrm{CTLA}_{4}$ are used to block their inhibitory action and further robust immune responses are carried out for durable period of time. ${ }^{39}$ In many cancers up regulation of $\mathrm{PD}_{1}$ and $\mathrm{CTLA}_{4}$ was observed in tumor infiltration lymphocytes along with higher $\mathrm{PD}-\mathrm{L}_{1}$ expression on cancer cells which serve to shield the cancer cells against immune attack by keeping $\mathrm{T}$ cell activity at basal levels. ${ }^{39}$ Especially, the triple negative breast cancer needs significant interactions with the microenvironment including stroma, tumor infiltrated lymphocytes, fibroblasts and macrophages as it is not hormonal dependent. These multiple interactions reflected in the tumor as mutations and clonal differences making the overall tumor resistant to treatment. ${ }^{40}$ Hence $\mathrm{PD}_{1} / \mathrm{CTLA}_{4}$ targeted therapy is highly advisable in cancer treatment. $\mathrm{PD}_{1}$ directed antibodies including brolizumab and nivolumab and $\mathrm{CTLA}_{4}$ targeted antibodies such as dacarbazine are already tested rigorously in against cancers for their efficacy. ${ }^{41}$

Combination treatment of ipilimimab and dacarbazine in phase 3 studies in previously untreated metastatic melanoma patients was conducted to see overall survival rate. ${ }^{42}$ Significant increase in overall survival was observed in patients receiving combination treatment when compared to dacarbazine and place treated group. 3 year overall survival was observed in $20.8 \%$ of patients while the control group has only $12.2 \%$ of overall survival rate. Moreover, the check point blockade antibodies can be combined with chemotherapeutic agents for improved efficacy. In another study, tremelimumab $\left(\right.$ CTLA $_{4}$ specific monoclonal antibody) was combined with exemestane (steroidal aromatase inhibitor) to test in hormone responsive breast cancer patients to check the maximum tolerance dose, safety and assess tumor response. ${ }^{43}$ The treatment resulted in mild to moderate levels of adverse events and increased peripheral $\mathrm{CD}_{4+}$ and $\mathrm{CD}_{8+} \mathrm{T}$ cells expressing inducible co-stimulator (ICOS). There was also an increased ratio of ICOS $+\mathrm{T}$ cells to $\mathrm{T}$ regulatory cells. This type of response can activate immune signals along with CTLA 4 blockade.

\section{Vitamins in cancer immunotherapy}

In a clinical trial, vitamin A was administered alone or in combination with chemotherapy or radiotherapy tested in clinical trial by administering alone or in combination with chemotherapy and radiotherapy in unrespectable bronchogenic cancer patients. ${ }^{44}$ Metastatic unrespectable squamous cell carcinoma of lung was treated in 9 patients with Vitamins A in a 60 weeks study. In addition, vitamin A was found to boost the patient immune system evident by increased lymphocyte blastogenesis response. ${ }^{44}$ This shows powerful anticancer activity of Vitamin A and its parallel immune boosting effect. Vitamin $\mathrm{E}$ is widely studies natural molecule in cancer treatment. Vitamin $\mathrm{E}$ is divided into tocopherols and tocotrienols. ${ }^{45}$ while the tocopherols exert mainly antioxidant properties, tocotrienols were proven for their potent anticancer activity against various cancer types in animal studies. The anticancer activities of vitamin $\mathrm{E}$ are mediated by apoptosis, autophagy, cell cycle inhibition, and metastasis inhibition pathways. ${ }^{45}$ Suppression of inflammatory pathway by inhibition of transcription factor $\mathrm{NF}-\mathrm{\kappa B}$, and further slower the tumor growth, and inhibition of HMG-CoA reductase enzyme responsible for protein prenylation, 
targeting DNA polymerases and protein tyrosine kinases are the few mechanisms mediated by tocotrienols. ${ }^{45}$ Besides, vitamin $\mathrm{E}$ was also proved for its immune boosting functions in recent studies. Vitamin $\mathrm{E}$ supplementation at a daily dose of $750 \mathrm{mg}$ for 2 weeks in patients with colorectal cancer resulted in increased $\mathrm{CD}_{4}: \mathrm{CD}_{8}$ ratio and enhanced $\mathrm{T}$ cell capacity to produce $\mathrm{T}$ helper 1 cytokine interleukin 2 and IFN $\gamma .{ }^{46}$ In a separate study, anti-oxidant vitamin E supplementation at daily dose of $750 \mathrm{mg}$ for 2 weeks increased NK cells' cytolytic activity in colorectal cancer patients. Vitamin E containing self-emulsifying nano-emulsion systems is used for drug delivery. ${ }^{47}$ When vitamin E nano emulsion was loaded with paclitaxel (PTX), it showed increased cytotoxicity against MCF-7 breast cancer cells compared to the PTX treatment alone. ${ }^{48}$ Vitamin E combination with PTX in the form of nano-emulsion not only aiding drug release at controlled rate but also has its own pharmacological activity by inducing apoptosis and growth arrest at $\mathrm{G}_{1}-\mathrm{S}$ stage of cell cycle progression. When PTX was loaded into the emulsion, growth arrest was induced in MCF-7 cells at $\mathrm{G}_{2}-\mathrm{M}$ phase, and increased IL -12 secretion from macrophages and down regulated IL -4 and IL -10 cytokine secretion were observed. Moreover, the PTX nano-emulsion has benefits with increased in-vivo anticancer activity, and associated with lower in vivo toxicity and altered pharmacokinetic profile AUC and MRT were extended. ${ }^{48,49}$ In sum, this shows the importance vitamin $\mathrm{E}$ in boosting the pharmacological and immunological responses when combined with PTX in breast cancer.

\section{Conclusion}

In sum, it is the time to develop immunotherapies against cancer to boost anticancer immunity. It is a challenge to study each and every patient's tumor type and further tumor microenvironment interactions. However, treatments can be developed to inhibit tumor infiltration of immune cells or inhibit their functionalization to support therapy and favour regression. As there is high clonal differences in immune cells, development of resistance against a specific treatment should also be considered. Understanding tumor cell antigen specific priming of $\mathrm{T}$ cells is one of the ideas to target. It is strongly advisable to prevent the cancer occurrence through food habits if possible.

\section{Acknowledgements}

None.

\section{Conflict of interest}

The author declares no conflict of interest.

\section{References}

1. Marrack P, Kappler JW. How the immune system recognizes the body. Sci Am. 1993;269(3):80-83.

2. Chandra RK. Nutrition and the immune system: an introduction. Am J Clin Nutr. 1997;66(2):460S-463S

3. de Visser KE, Eichten A, Coussens LM. Paradoxical roles of the immune system during cancer development. Nat Rev Cancer. 2006;6(1):24-37.

4. Ananthula S, Sinha A, Gassim M, et al. Geminin overexpressiondependent recruitment and crosstalk with mesenchymal stem cells enhance aggressiveness in triple negative breast cancers. Oncotarget. 2016;7(15):20869-20889.

5. Hosain SB, Khiste SK, Uddin MB, et al. Inhibition of glucosylceramide synthase eliminates the oncogenic function of p53 R273H mutant in the epithelial-mesenchymal transition and induced pluripotency of colon cancer cells. Oncotarget. 2016;7(37):60575-60592.
6. Denny WA. Prodrug strategies in cancer therapy. Eur J Med Chem. 2001;36(8):577-595.

7. Kerbel RS. A cancer therapy resistant to resistance. Nature. 1997;390(6658):335-336.

8. Horvat TZ, Adel NG, Dang TO, et al. Immune-related adverse events, need for systemic Immunosuppression and effects on survival and time to treatment failure in patients with melanoma treated with Ipilimumab at Memorial Sloan Kettering Cancer Center. J Clin Oncol. 2015;33(28):3193-3198.

9. Gajewski TF, Schreiber H, Fu YX. Innate and adaptive immune cells in the tumor microenvironment. Nat Immunol. 2013;14(10):1014-1022.

10. Joyce JA, Fearon DT. T cell exclusion, immune privilege, and the tumor microenvironment. Science. 2015;348(6230):74-80.

11. Bissell MJ, Labarge MA. Context, tissue plasticity, and cancer: are tumor stem cells also regulated by the microenvironment? Cancer Cell. 2005;7(1):17-23.

12. Ostrand-Rosenberg S, Sinha P. Myeloid-derived suppressor cells: linking inflammation and cancer. J Immunol. 2009;182(8):4499-4506.

13. Chen Q, Zhang XH, Massagué J. Macrophage binding to receptor VCAM-1 transmits survival signals in breast cancer cells that invade the lungs. Cancer cell. 2011;20(4):538-549.

14. Chen Q, Massagué J. Molecular pathways: VCAM-1 as a potential therapeutic target in metastasis. Clin Cancer Res. 2012;18(20):5520-5525.

15. Mantovani A, Schioppa T, Porta C, et al. Role of tumor-associated macrophages in tumor progression and invasion. Cancer Metastasis Rev. 2006;25(3):315-322.

16. Pardoll DM. The blockade of immune checkpoints in cancer immunotherapy. Nat Rev Cancer. 2012;12(4):252-264.

17. Couzin-Frankel J. Cancer immunotherapy. Science. 2013;342(6165):1432-1433.

18. Schumacher TN, Schreiber RD. Neoantigens in cancer immunotherapy. Science. 2015;348(6230):69-74.

19. Nobili S, Lippi D, Witort E, et al. Natural compounds for cancer treatment and prevention. Pharmacol Res. 2009;59(6):365-378.

20. Ananthula S, Parajuli P, Behery FA, et al. Oxazine derivatives of $\gamma$-and $\delta$-tocotrienol display enhanced anticancer activity in vivo. Anticancer Res. 2014;34(6):2715-2726.

21. Ananthula S, Parajuli P, Behery FA, et al. $\delta$-Tocotrienol oxazine derivative antagonizes mammary tumor cell compensatory response to $\mathrm{CoCl}_{2}-$ induced hypoxia. Biomed Res Int. 2014;2014:285752.

22. Amin AR, Kucuk O, Khuri FR, et al. Perspectives for cancer prevention with natural compounds. J Clin Oncol. 2009;27(16):2712-2725.

23. Yamamoto N, Suyama H, Yamamoto N, et al. Retracted: Immunotherapy of metastatic breast cancer patients with vitamin D-binding protein-derived macrophage activating factor (GcMAF). Int $J$ Cancer. 2008;122(2):461-467.

24. Malmberg KJ. Effective immunotherapy against cancer. Cancer Immunol Immunother. 2004;53(10):879-892.

25. Pardoll DM. Cancer vaccines. Nature medicine. 1998;(4):525-531.

26. Disis ML, Schiffman K. Cancer vaccines targeting the $\mathrm{HER}_{2} /$ neu oncogenic protein. Semin Oncol. 2001;28(18):12-20.

27. Mittendorf EA, Clifton GT, Holmes JP, et al. Final report of the phase I/ II clinical trial of the $\mathrm{E}_{75}$ (nelipepimut-S) vaccine with booster inoculations to prevent disease recurrence in high-risk breast cancer patients. Ann Oncol. 2014;25(9):1735-1742. 
28. Holmes JP, Benavides LC, Gates JD, et al. Results of the first phase I clinical trial of the novel II-key hybrid preventive HER-2/neu peptide $\left(\mathrm{AE}_{37}\right)$ vaccine. J Clin Oncol. 2008;26(20):3426-3433.

29. Banchereau J, Palucka AK. Dendritic cells as therapeutic vaccines against cancer. Nat Rev Immunol. 2005;5(4):296-306.

30. Jefford M, Maraskovsky E, Cebon J, et al. The use of dendritic cells in cancer therapy. The lancet oncology. 2001;2(6):343-353.

31. Ladjemi MZ, Jacot W, Chardès $T$, et al. Anti-HER vaccines: new prospects for breast cancer therapy. Cancer Immunol Immunother. 2010;59(9):1295-1312.

32. ML Disis, MM OLMeara. HER 2 /neu-23. Cancer Therapeutic Targets. 2017. 267 p.

33. Mitchell PL, Quinn MA, Grant PT, et al. A phase 2, single-arm study of an autologous dendritic cell treatment against mucin 1 in patients with advanced epithelial ovarian cancer. J Immunother Cancer. 2014;2(1):16.

34. Svane IM, Pedersen AE, Johansen JS, et al. Vaccination with P53 peptide-pulsed dendritic cells is associated with disease stabilization in patients with $\mathrm{P}_{53}$ expressing advanced breast cancer; monitoring of serum YKL -40 and IL-6 as response biomarkers. Cancer Immunol Immunother. 2007;56(9):1485-1499.

35. Hosain SB, YY Liu. Abstract 5191: Missense mutants of $\mathrm{P}_{53}$ tumor suppressor contributes to drug-resistance and epithelial-mesenchymal transition in colon cancer cells. AACR. 2015;77(15 Suppl).

36. Chen L. Co-inhibitory molecules of the $\mathrm{B}_{7}-\mathrm{CD}_{28}$ family in the contro of T-cell immunity. Nat Rev Immunol. 2004;4(5):336-347.

37. Nishimura H, Honjo T. PD-1: an inhibitory immunoreceptor involved in peripheral tolerance. Trends Immunol. 2001;22(5):265-268.

38. Wing K, Onishi Y, Prieto-Martin P, et al. CTLA-4 control over Foxp3+ regulatory T cell function. Science. 2008;322(5899):271-275.

39. Topalian SL, Drake CG, Pardoll DM. Immune checkpoint blockade: a common denominator approach to cancer therapy. Cancer cell. 2015;27(4):450-461.

40. Greaves M, Maley CC. Clonal evolution in cancer. Nature. 2012;481(7381):306-313
41. Callahan MK, Wolchok JD. At the bedside: CTLA-4-and PD-1blocking antibodies in cancer immunotherapy. $J$ Leukoc Biol. 2013;94(1):41-53.

42. Maio M, Grob JJ, Aamdal S, et al. Five-year survival rates for treatment-naive patients with advanced melanoma who received ipilimumab plus dacarbazine in a phase III trial. J Clin Oncol. 2015;33(10):11911196.

43. Vonderheide RH, LoRusso PM, Khalil M, et al. Tremelimumab in combination with exemestane in patients with advanced breast cancer and treatment-associated modulation of inducible co-stimulator expression on patient T cells. Clin Cancer Res. 2010;16(13):3485-3494.

44. Micksche M, Cerni C, Kokron O, et al. Stimulation of immune response in lung cancer patients by vitamin A therapy. Oncology. 1977;34(5):234 238.

45. Aggarwal BB, Sundaram C, Prasad S, et al. Tocotrienols, the vitamin E of the $21^{\text {st }}$ century: its potential against cancer and other chronic diseases. Biochem Pharmacol. 2010;80(11):1613-1631.

46. Malmberg KJ, Lenkei R, Petersson M, et al. A short-term dietary supplementation of high doses of vitamin $\mathrm{E}$ increases T helper 1 cytokine production in patients with advanced colorectal cancer. Clin Cancer Res. 2002;8(6):1772-1778.

47. Hanson MG, Ozenci V, Carlsten MC, et al. A short-term dietary supplementation with high doses of vitamin E increases NK cell cytolytic activity in advanced colorectal cancer patients. Cancer Immunol Immunother. 2007;56(7):973-984

48. Pawar VK, Panchal SB, Singh Y, et al. Immunotherapeutic vitamin E nanoemulsion synergies the antiproliferative activity of paclitaxel in breast cancer cells via modulating $\mathrm{Th}_{1}$ and $\mathrm{Th}_{2}$ immune response. $J$ Control Release. 2014;196:295-306.

49. Peethambaram PP, Melisko ME, Rinn KJ, et al. A phase I trial of immunotherapy with lapuleucel-T (APC8024) in patients with refractory metastatic tumors that express HER-2/neu. Clin Cancer Res. 2009;15(18):5937-5944. 